\title{
Intensive Care Risk Estimation in COVID-19 Pneumonia Based on Clinical and Imaging Parameters: Experiences from the Munich Cohort
}

\author{
Egon Burian ${ }^{1,2,+}{ }^{\oplus}$, Friederike Jungmann ${ }^{1,+}$, Georgios A. Kaissis ${ }^{1,+}{ }^{+}$, Fabian K. Lohöfer ${ }^{1,+}$, \\ Christoph D. Spinner ${ }^{3}{ }^{\circ}$, Tobias Lahmer ${ }^{3}$, Matthias Treiber ${ }^{3}$, Michael Dommasch ${ }^{4}$, \\ Gerhard Schneider ${ }^{5}$, Fabian Geisler ${ }^{3}$ D , Wolfgang Huber ${ }^{3}$, Ulrike Protzer ${ }^{6}$, Roland M. Schmid ${ }^{3}$, \\ Markus Schwaiger ${ }^{7}$, Marcus R. Makowski ${ }^{1, \ddagger}$ and Rickmer F. Braren ${ }^{1, *, \ddagger}$ \\ 1 Department of Diagnostic and Interventional Radiology, School of Medicine, Technical University of Munich, \\ Ismaninger Str. 22, 81675 Munich, Germany; egon.burian@tum.de (E.B.); friederike.jungmann@tum.de (F.J.); \\ g.kaissis@tum.de (G.A.K.); fabian.lohoefer@tum.de (F.K.L.); marcus.makowski@tum.de (M.R.M.) \\ 2 Department of Diagnostic and Interventional Neuroradiology, School of Medicine, \\ Technical University of Munich, Ismaninger Str. 22, 81675 Munich, Germany \\ 3 Department of Internal Medicine II, School of Medicine, Technical University of Munich, Ismaninger Str. 22, \\ 81675 Munich, Germany; Christoph.spinner@tum.de (C.D.S.); tobias.lahmer@mri.tum.de (T.L.); \\ Matthias.treiber@mri.tum.de (M.T.); fabian.geisler@tum.de (F.G.); wolfgang.huber@tum.de (W.H.); \\ direktion.med2@mri.tum.de (R.M.S) \\ 4 Department of Internal Medicine I, School of Medicine, Technical University of Munich, Ismaninger Str. 22, \\ 81675 Munich, Germany; Michael.dommasch@tum.de \\ 5 Clinic for Anesthesiology and Intensive Care Medicine, School of Medicine, Technical University of Munich, \\ Ismaninger Str. 22, 81675 Munich, Germany; Gerhard.schneider@tum.de \\ 6 Institute of Virology, School of Medicine, Technical University of Munich, Ismaninger Str. 22, \\ 81675 Munich, Germany; protzer@tum.de \\ 7 School of Medicine, Dean, Technical University of Munich, Ismaninger Str. 22, 81675 Munich, Germany; \\ markus.schwaiger@tum.de \\ * Correspondence: rbraren@tum.de; Tel.: +49-4140-5627 \\ + These authors contributed equally to this work. \\ $\ddagger$ These authors contributed equally to this work.
}

Received: 20 April 2020; Accepted: 15 May 2020; Published: 18 May 2020

\begin{abstract}
The evolving dynamics of coronavirus disease 2019 (COVID-19) and the increasing infection numbers require diagnostic tools to identify patients at high risk for a severe disease course. Here we evaluate clinical and imaging parameters for estimating the need of intensive care unit (ICU) treatment. We collected clinical, laboratory and imaging data from 65 patients with confirmed COVID-19 infection based on polymerase chain reaction (PCR) testing. Two radiologists evaluated the severity of findings in computed tomography (CT) images on a scale from 1 (no characteristic signs of COVID-19) to 5 (confluent ground glass opacities in over 50\% of the lung parenchyma). The volume of affected lung was quantified using commercially available software. Machine learning modelling was performed to estimate the risk for ICU treatment. Patients with a severe course of COVID-19 had significantly increased interleukin (IL)-6, C-reactive protein (CRP), and leukocyte counts and significantly decreased lymphocyte counts. The radiological severity grading was significantly increased in ICU patients. Multivariate random forest modelling showed a mean \pm standard deviation sensitivity, specificity and accuracy of $0.72 \pm 0.1,0.86 \pm 0.16$ and $0.80 \pm 0.1$ and a receiver operating characteristic-area under curve (ROC-AUC) of $0.79 \pm 0.1$. The need for ICU treatment is independently associated with affected lung volume, radiological severity score, CRP, and IL-6.
\end{abstract}

Keywords: COVID-19; severe acute respiratory syndrome coronavirus 2 (SARS-CoV-2); clinical parameters; radiological parameters; computed tomography; intensive care unit 


\section{Introduction}

At the end of 2019, infections with the novel severe acute respiratory syndrome coronavirus 2 (SARS-CoV-2) were first noted in Wuhan, China and rapidly spread to the rest of the world [1]. The lack of specific vaccines and treatments led to high infection and death counts around the world and thus caused a serious burden on national health care systems [2].

Even in highly developed health care systems, such as in Germany, with a very active testing and containment strategy alongside a federally guided preparation of the health care system for the coronavirus disease 2019 (COVID-19) pandemic, specific challenges are faced: pharyngeal swab-based polymerase chain reaction (PCR) testing is only highly sensitive during the early phase of infection and may miss the pulmonary phase of disease. Furthermore, limited resources both with respect to testing reagents and intensive care unit (ICU) capacities warrant identification of patients with primarily pulmonary disease manifestation at high risk for a severe course. Computed tomography (CT) was assigned a central role in patient stratification both in China and in Europe because of the high sensitivity, availability and speed [3-5]. Multiple studies have summarized clinical and CT imaging findings of COVID-19 pneumonia [6-8]. Typical imaging findings include confluent ground glass opacities, consolidation and crazy-paving patterns. More severe COVID-19 cases are frequently accompanied by laboratory and immunologic changes like C-reactive protein increases, lymphopenia and interleukin (IL)-6 peaks [9]. It stands to reason that the integration of imaging and clinical data can aid in the prediction of a severe course of disease in COVID-19 pneumonia and aid in patient stratification and triage, critical especially in an overloaded health care system as was observed in Italy [2] and more recently, New York [10].

Here we present the results of the integrated analysis of the first large scale outbreak in Southern Germany, Munich, exemplified by a cohort of 65 consecutive patients admitted to the emergency department and requiring hospitalization, and identify pertinent risk factors for ICU treatment.

\section{Experimental Section}

\subsection{Patients}

This study was conducted according to the principles set forward in the Declaration of Helsinki and according to Good Clinical Practice. All patients gave consent for scientific evaluation of clinical and imaging data at the time of admission. The local institutional review board of the Technical University of Munich has approved this prospective study (protocol numbers: 245/19 S-SR and 111/20 S). The study was designed as a retrospective cohort study. The STROBE checklist and patient recruitment flowchart (Figure S1) are included in the Supplementary Material [11].

Sixty-five consecutive patients were included in this study according to the Diagnosis and Treatment of Novel Coronavirus Pneumonia (5th version) of China [12], who presented in our hospital between March and April 2020. Inclusion criteria were defined as follows: Positive real-time reverse-transcriptase polymerase-chain-reaction (RT-PCR) testing for SARS-CoV-2 nucleic acid in throat swabs or lower respiratory tract lavage, characteristic imaging findings in thin-section CT, and moderate to severe symptoms (fever, dyspnea and/or dry cough) requiring hospitalization. Patients who developed one or more of the following symptoms were admitted to the ICU: respiratory rate $\geq 30$ breaths per minute, peripheral resting state oxygen saturation $\leq 93 \%$, invasively measured arterial oxygen tension $\left(\mathrm{P}_{\mathrm{a}} \mathrm{O}_{2}\right) /$ inspiratory oxygen fraction $\left(\mathrm{F}_{\mathrm{i}} \mathrm{O}_{2}\right) \leq 300 \mathrm{mmHg}(1 \mathrm{mmHg}=0.133 \mathrm{kPa})$, respiratory failure requiring mechanical ventilation, cardiovascular shock, and/or miscellaneous organ failure. Patients with a negative RT-PCR were excluded from the analyses. 
The following patient medical record items were collected: comorbidities (systemic hypertension, diabetes mellitus, heart disease and chronic obstructive or restrictive pulmonary disease), medical history, and physical examination. Routine laboratory tests were performed including complete blood count and serum biochemistry (leukocytes, lymphocytes, creatinine, creatine kinase, creatine kinase myocardial band (CK-MB), C-reactive protein (CRP), troponin-T, lactate dehydrogenase (LDH), D-dimer, interleukin 6 (IL-6)).

\subsection{MDCT Imaging}

All patients were examined with the same 256-row multidetector computed tomography (MDCT) scanner (iCT, Philips Healthcare, Best, The Netherlands). Pulmonary MDCT was performed with the following parameters in full inspiration with arms elevated and no administration of contrast agent. One-hundred-and-twenty peak kilovoltage tube voltage, adapted tube load of averaged $200 \mathrm{mAs}$ and minimum collimation $(0.6 \mathrm{~mm})$ were used. The mean dose length product was $217 \pm 270 \mathrm{mGy} / \mathrm{cm}$.

\subsection{Severity Score in $\mathrm{CT}$}

Two radiologists with 8 (F.K.L.) and 3 years of experience (E.B.) performed qualitative image assessment blinded to the clinical data. For each of the 65 patients, chest CTs were evaluated according to the criteria presented in Figure 1. The spectrum of imaging manifestations ranged from grade 1 to 5 . The intraclass correlation coefficient was calculated between the assessments.
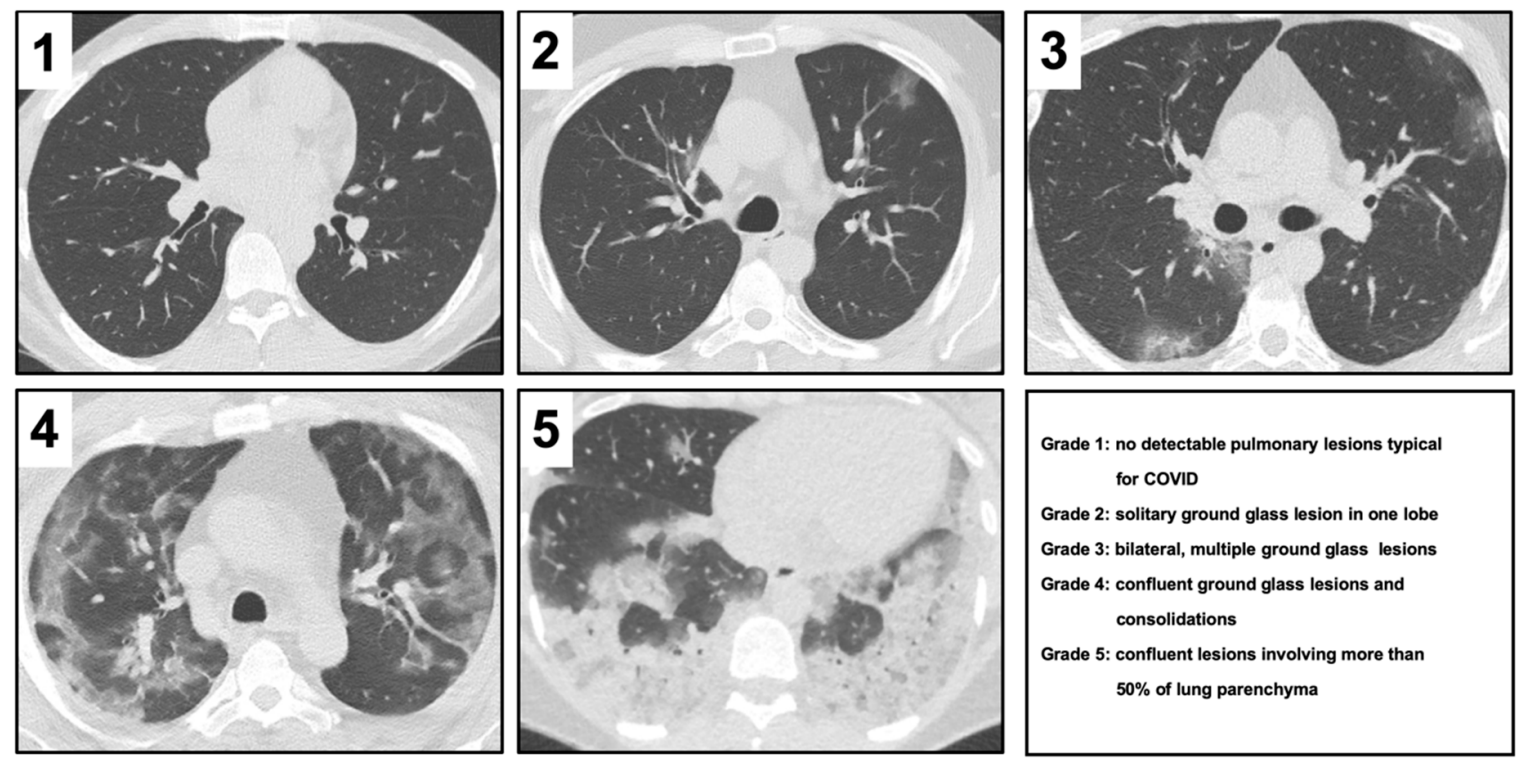

Figure 1. Images exemplifying the five radiological severity scores in computed tomography images alongside their descriptions. COVID, coronavirus disease.

\subsection{Semi-Automated Lung Parenchyma Volume Quantification}

$\mathrm{CT}$ datasets were semi-automatically segmented, and segmentations were manually corrected (IntelliSpace Portal 11, Philips Healthcare, Best, The Netherlands). Briefly, the complete lung volume and the volume of each lobe was quantified. Then, ground glass opacifications and consolidations were subtracted from the measured volume based on expert-determined threshold adaption, within the range of $-600 \mathrm{HU}$ to $-770 \mathrm{HU}$ (Figures 2 and 3). 

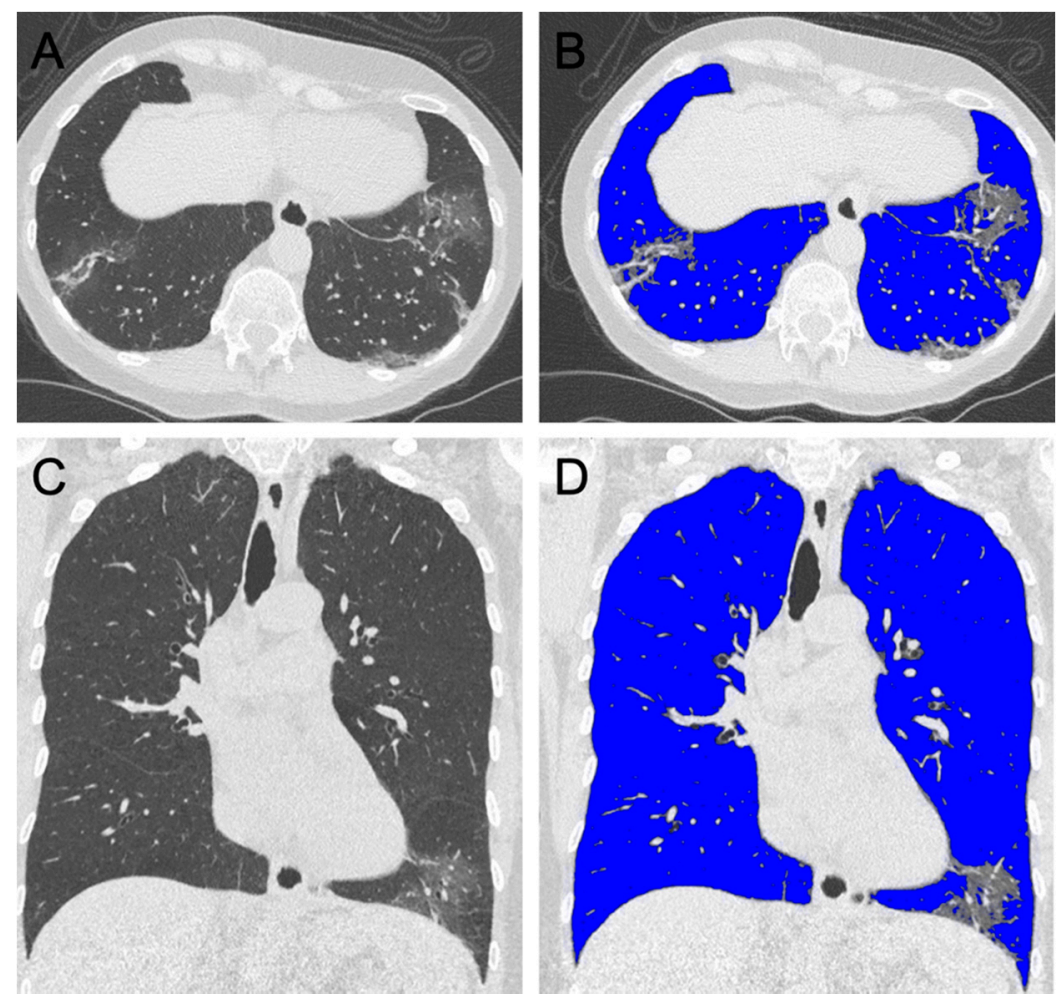

Figure 2. Axial (A,B) and coronal (C,D) reformations of the chest computed tomography (CT) of a 68 -year-old female patient presenting with fever and cough. Radiological severity grade 3 was assigned. Lung volume quantification accounted for $>90 \%$ ventilated volume (shaded blue in B and D).
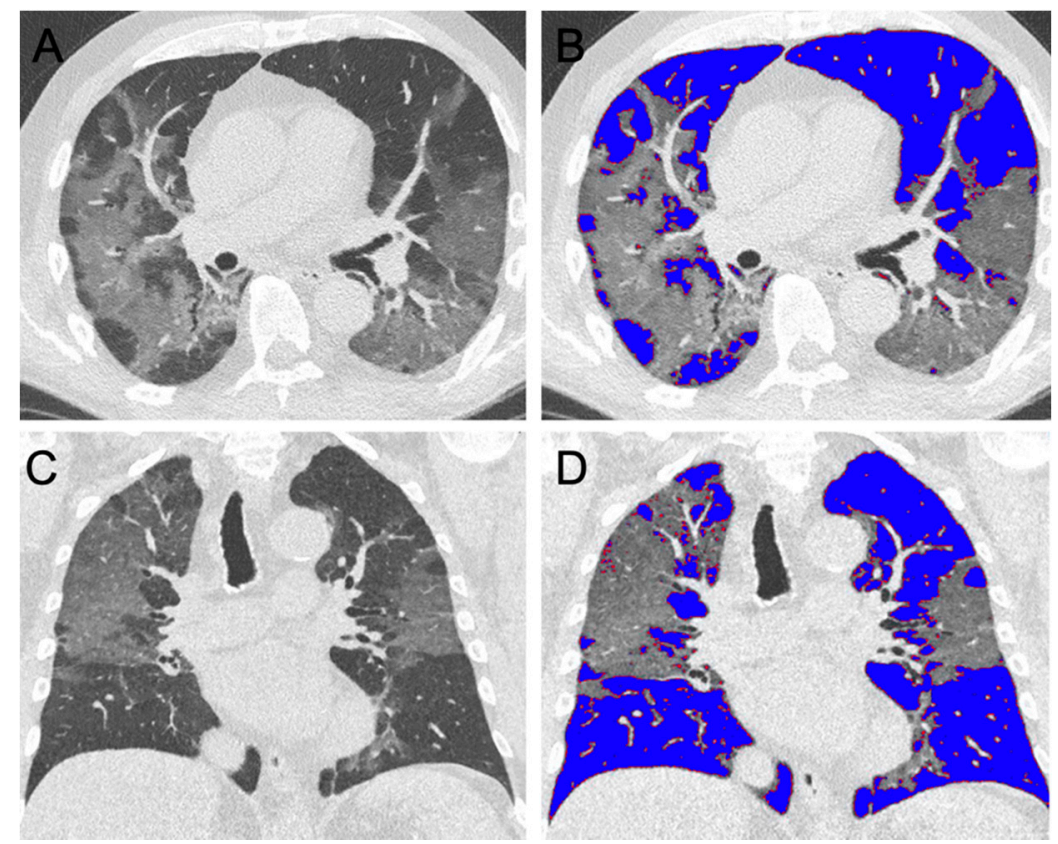

Figure 3. Axial (A,B) and coronal (C,D) reformations of the chest CT of a 78-year-old-male patient presenting with mild dyspnea. Radiological severity grade 4 was assigned. Forty-five percent of the lung volume is affected by characteristic parenchymal changes, most prominent in the right upper lobe (shaded blue in $\mathbf{B}$ and $\mathbf{D}$ ). 


\subsection{Statistical Analysis}

Statistical analyses were performed in SPSS (version 26; SPSS Inc., Chicago, IL, USA) and Python 3.7.6. All tests were carried out using two-sided 0.05 level of significance. For multivariate random forest modelling, the proportion of ventilated lung parenchyma was computed for upper and lower lung from the semi-automated volume quantification. The middle lobe of the right lung was attributed to the upper lung. As the severity scores determined by the two radiologists had a high concordance, their average score was used in the model. All parameters were normalized to the unity interval. Single missing values were imputed using the mean value of continuous features or the mode in case of binary features. The random forest model was fit to all clinical and radiological data to predict the necessity of ICU treatment. Five-fold cross validation was performed with $80 \%$ of the data used for training and $20 \%$ for testing. Mean \pm standard deviation (SD) importance for the features and the performance of the random forest model was assessed by cross validation.

\section{Results}

Sixty-five patients ( 23 female, 42 male, $61.5 \pm 17.0$ years) were included in this study. The most common symptoms were fever, cough, dyspnea, and gastrointestinal symptoms. The condition of 28 ( 6 female, 22 male, $64.9 \pm 16.6$ years, range $28-97$ years) of these patients was deteriorating and necessitated ICU treatment. The clinical and laboratory parameters of the presented cohort are summarized in Table 1. The imaging-based severity assessment and volumetric lung parenchyma analysis is shown in Table 2. The analysis of the blood samples revealed a significant increase of leukocyte counts, CRP and IL-6 $(p<0.0001)$ and a significant reduction of the lymphocyte count in ICU patients $(p<0.0001)$ (Table 1$)$.

Table 1. Descriptive statistics of the clinical and laboratory parameters. Parameters were compared between the two groups with student's $t$ test.

\begin{tabular}{|c|c|c|c|c|c|c|c|}
\hline & \multicolumn{3}{|c|}{ Mitigated Group } & \multicolumn{3}{|c|}{ ICU Group } & \multirow[b]{2}{*}{$p$} \\
\hline & $n$ & Mean & SD & $n$ & Mean & SD & \\
\hline Age (years) & 37 & 59.0 & 17.1 & 28 & 64.9 & 16.6 & 0.170 \\
\hline $\begin{array}{l}\text { Percutaneous oxygen } \\
\text { saturation }(\%)\end{array}$ & 32 & 95.9 & 2.6 & 20 & 91.5 & 8.3 & 0.007 \\
\hline Temperature $\left({ }^{\circ} \mathrm{C}\right)$ & 37 & 37.8 & 0.9 & 21 & 37.6 & 17.9 & 0.173 \\
\hline Leucocytes (G/L) & 37 & 5.6 & 2.5 & 28 & 8.2 & 4.0 & 0.002 \\
\hline Lymphocytes (\%) & 37 & 29.1 & 49.6 & 25 & 26.2 & 64.6 & $<0.001$ \\
\hline CRP (mg/dL) & 37 & 4.8 & 6.3 & 28 & 12.4 & 9.7 & $<0.001$ \\
\hline Creatinine $(\mathrm{mg} / \mathrm{dL})$ & 37 & 1.3 & 1.9 & 28 & 1.4 & 0.7 & 0.855 \\
\hline D-dimer $(\mu \mathrm{g} / \mathrm{mL})$ & 31 & 1631.9 & 2573.3 & 18 & 2407.2 & 2856.5 & 0.334 \\
\hline $\mathrm{LDH}(\mathrm{U} / \mathrm{L})$ & 35 & 394.1 & 529.3 & 24 & 487.6 & 277.7 & 0.431 \\
\hline Creatine kinase (U/L) & 34 & 153.91 & 165.2 & 24 & 632.4 & 1622.1 & 0.092 \\
\hline $\mathrm{CK}-\mathrm{MB}(\mathrm{U} / \mathrm{L})$ & 1 & 37.0 & 32.4 & 5 & 42.8 & 25.3 & \\
\hline Troponin-T (ng/mL) & 7 & 0.3 & 0.7 & 8 & 0.1 & 0.1 & 0.499 \\
\hline IL-6 (pg/mL) & 25 & 51.7 & 65.6 & 12 & 103.9 & 43.6 & 0.017 \\
\hline
\end{tabular}

ICU, intensive care unit; $\mathrm{SD}$, standard deviation; $\mathrm{CRP}, \mathrm{C}$-reactive protein; $\mathrm{LDH}$, lactate dehydrogenase; IL, interleukin; CK-MB, creatine kinase myocardial band.

The Cohen's kappa testing showed excellent agreement in CT-based severity score rating between the two radiologists $(\kappa=0.81)$. Lung volume quantification revealed a significant increase in the percentage of opacifications in ICU patients $(41.6 \%)$ compared to non-ICU patients $(19.3 \%$, $t$-test $p<0.001$ ).

Five-fold cross validation of the Random Forest model yielded a mean \pm SD sensitivity of $0.72 \pm 0.1$, specificity of $0.86 \pm 0.16$, accuracy of $0.80 \pm 0.1$ and ROC-AUC of $0.79 \pm 0.1$. The five most important features for classification were ventilation of the upper lung $(0.184 \pm 0.025)$, ventilation of the lower lung $(0.123 \pm 0.03), \mathrm{CRP}(0.074 \pm 0.018)$, radiological severity score $(0.067 \pm 0.011)$, and IL-6 (0.058 \pm 0.006$)$. 
The five most important features are displayed in Table 3. Imaging parameters alone resulted in a mean \pm SD sensitivity of $0.78 \pm 0.2$, specificity of $0.78 \pm 0.11$, accuracy of $0.78 \pm 0.1$, and ROC-AUC of $0.79 \pm 0.12$. The addition of CRP to the imaging parameters resulted in a mean \pm SD sensitivity of $0.76 \pm 0.08$, specificity of $0.81 \pm 0.13$, accuracy of $0.78 \pm 0.06$, and ROC-AUC of $0.79 \pm 0.07$. Finally, the addition of IL- 6 to imaging parameters and CRP resulted in a mean \pm SD sensitivity of $0.76 \pm 0.08$, specificity of $0.81 \pm 0.13$, accuracy of $0.78 \pm 0.08$, and ROC-AUC of $0.79 \pm 0.07$.

Table 2. Descriptive statistics of the imaging parameters. Parameters were compared between the two groups with student's $\mathrm{t}$ test.

\begin{tabular}{cccccccc}
\hline & \multicolumn{3}{c}{ Mitigated Group } & \multicolumn{7}{c}{ ICU Group } \\
\hline & $n$ & Mean & SD & $n$ & Mean & SD & $p$ \\
\hline CT severity score & 37 & 2.9 & 0.9 & 28 & 4.0 & 1.0 & $<0.001$ \\
Lung ventilated (\%) & 37 & 80.7 & 11.2 & 27 & 58.4 & 15.9 & $<0.001$ \\
Right lung (\%) & 37 & 80.7 & 10.7 & 27 & 57.0 & 17.6 & $<0.001$ \\
Right upper lobe (\%) & 37 & 83.1 & 12.4 & 27 & 56.4 & 21.7 & $<0.001$ \\
Right middle lobe (\%) & 37 & 88.0 & 7.4 & 27 & 71.0 & 17.4 & $<0.001$ \\
Right lower lobe (\%) & 37 & 74.5 & 13.9 & 27 & 49.4 & 20.2 & $<0.001$ \\
Left lung (\%) & 37 & 80.5 & 12.6 & 27 & 58.8 & 17.2 & $<0.001$ \\
Left upper lobe (\%) & 37 & 85.5 & 10.3 & 27 & 62.9 & 17.0 & $<0.001$ \\
Left lower lobe (\%) & 37 & 74.1 & 16.9 & 27 & 52.2 & 21.5 & $<0.001$ \\
\hline
\end{tabular}

$\mathrm{CT}$, computed tomography.

Table 3. Mean \pm SD of random forest classification feature importance across the cross-validation folds.

\begin{tabular}{ccc}
\hline Feature & Mean Importance & Standard Deviation \\
\hline Ventilation upper lung & 0.184 & 0.025 \\
Ventilation lower lung & 0.123 & 0.030 \\
CRP & 0.074 & 0.018 \\
Radiological Severity Score & 0.067 & 0.011 \\
IL-6 & 0.058 & 0.006 \\
\hline
\end{tabular}

\section{Discussion}

We here present the multivariate analysis of clinical and imaging data of a cohort of 65 related to the first large scale outbreak of COVID-19 in Southern Germany. We identify imaging derived features (upper and lower lung opacifications, radiological severity estimation), CRP and IL-6 levels as the most important features for the prediction of the necessity of ICU admission. Our results underline the importance of the integration of CT imaging into the management of symptomatic COVID-19 pneumonia patients during this pandemic.

During the course of the COVID-19 pandemic, data related to epidemiological $[13,14]$, clinical [15], virological [16], immunological [9], and imaging findings [6,17] of COVID-19, and their interdependence [18] is rapidly accumulating. In light of limited capacities and resources, with respect to ICU treatment, personal protective equipment and personnel [19], informed patient management is essential for planning. Even highly developed health care systems, such as the Italian, were faced with an unprecedented shortage of resources leading to the implementation of triage systems for the allocation of hospital and mechanical ventilation capacities [20].

Several current studies have correlated biomarkers and imaging derived markers with a severe disease course [21]. However, so far, to our knowledge, no studies have investigated markers for the prediction of the necessity of ICU treatment.

Our data indicate affection of the upper lung lobes as an easily identifiable and highly important predictive parameter of ICU treatment. This is consistent with the typical finding of COVID-19 pneumonia, which was often noted in the lower peripheral lobes of the lung, presumably involving the 
upper lobes in more advanced cases. This result, alongside the semi-quantitative severity score shows that simple metrics of pulmonary involvement can be used to predict clinical outcome. It remains unknown at this time how these imaging features relate to the individuals' viral load. However, it is assumed that SARS CoV2 replicates in the lung tissue and it has been postulated that a failure to reduce viral load in the lung correlates with worse clinical outcome, which in turn is associated with more severe imaging findings [22,23]. Despite recent advances in molecular viral characterization [24], the recent evidence does not support a severity or mortality stratification based on viral load dynamics. However, the presented multiparametric severity assessment (including IL-6 and CRP) showed excellent correlation with disease severity and individuals' risk for an aggravated course of disease.

Previous studies have addressed disease severity assessment in infectious lung diseases including qualitative and semi-quantitative approaches $[25,26]$. In these studies, imaging findings were also associated with higher mortality $[25,26]$. Our study confirms these findings and holds the additional benefit of a simplified image analysis and the integration of multiparametric patient data.

Although the presented data suggests the possibility of an estimation of disease severity in COVID-19 pneumonia using broadly available and simply assessed parameters, there are some limitations to our findings. The main limitation of our study is the lack of an external validation cohort. Further, the cohort size is small and therefore the importance of comorbidities is likely underrepresented in our analysis. Despite this, none of the ICU admitted patients seemed to suffer a clinical course defined by their comorbidity profile. Furthermore, in our current analysis we did not distinguish between different types of opacification (i.e., ground glass versus consolidated volumes) nor between clinically defined early and late stage manifestations of COVID-19 pneumonia. These may hold differential sensitivity for predicting the clinical course of disease and would first require validation in longitudinal imaging studies. Clearly, at this point we can only speculate on the clinical value of CT imaging for the prediction of individual viral load as other factors (e.g., host genetics and immune system interplay) may play in important role.

\section{Conclusions}

Our study presents CT imaging-derived findings and serum markers of inflammation as best suited predictors of the necessity for ICU admission of symptomatic COVID-19 pneumonia patients. We encourage the prospective validation of these results in clinical patient management.

Supplementary Materials: The following are available online at http://www.mdpi.com/2077-0383/9/5/1514/s1, Figure S1: STROBE checklist and patient recruitment flow chart.

Author Contributions: E.B.: data acquisition, data post-processing, statistical analysis, drafting of manuscript, F.J.: data post-processing, statistical analysis, data acquisition, critical revision of manuscript, G.A.K.: data post-processing, statistical analysis, critical revision of manuscript, F.K.L.: data post-processing, critical revision of manuscript, C.D.S.: concept of study design, data acquisition, critical revision of manuscript, T.L.: critical revision of manuscript, M.T.: critical revision of manuscript, M.D.: critical revision of manuscript, G.S.: critical revision of manuscript, W.H.: concept of study design, data acquisition, critical revision of manuscript, F.G.: concept of study design, data acquisition, critical revision of manuscript, U.P.: concept of study design, data acquisition, critical revision of manuscript, R.M.S.: concept of study design, data acquisition, critical revision of manuscript, M.S.: concept of study design, data acquisition, critical revision of manuscript, M.R.M.: concept of study design, data acquisition, data post-processing, statistical analysis, critical revision of manuscript, R.F.B.: concept of study design, data acquisition, data post-processing, statistical analysis, critical revision of manuscript. All authors have read and agreed to the published version of the manuscript.

Conflicts of Interest: The authors declare no conflict of interest.

\section{References}

1. Coronavirus Disease 2019 (COVID-19)—Situation Report-44. Available online: https://www.who.int/docs/ default-source/coronaviruse/situation-reports/20200304-sitrep-44-covid-19.pdf?sfvrsn=93937f92_6 (accessed on 4 March 2020).

2. Rosenbaum, L. Facing Covid-19 in Italy-ethics, logistics, and therapeutics on the epidemic's front line. N. Engl. J. Med. 2020, 382, 1873-1875. [CrossRef] [PubMed] 
3. Ye, Z.; Zhang, Y.; Wang, Y.; Huang, Z.; Song, B. Chest CT manifestations of new coronavirus disease 2019 (COVID-19): A pictorial review. Eur. Radiol. 2020. [CrossRef] [PubMed]

4. Bernheim, A.; Mei, X.; Huang, M.; Yang, Y.; Fayad, A.Z.; Zhang, N.; Diao, K.; Lin, B.; Zhu, X.; Li, K. Chest CT findings in Coronavirus disease-19 (COVID-19): Relationship to duration of infection. Radiology 2020, 200463. [CrossRef] [PubMed]

5. Zhou, Z.; Guo, D.; Li, C.; Fang, Z.; Chen, L.; Yang, R.; Li, X.; Zeng, W. Coronavirus disease 2019: Initial chest CT findings. Eur. Radiol. 2020. [CrossRef] [PubMed]

6. Li, K.; Fang, Y.; Li, W.; Pan, C.; Qin, P.; Zhong, Y.; Liu, X.; Huang, M.; Liao, Y.; Li, S. CT image visual quantitative evaluation and clinical classification of coronavirus disease (COVID-19). Eur. Radiol. 2020. [CrossRef]

7. Wong, H.Y.F.; Lam, H.Y.S.; Fong, A.H.-T.; Leung, S.T.; Chin, T.W.-Y.; Lo, C.S.Y.; Lui, M.M.-S.; Lee, J.C.Y.; Chiu, K.W.-H.; Chung, T. Frequency and distribution of chest radiographic findings in COVID-19 positive patients. Radiology 2019, 201160. [CrossRef]

8. Xiong, Y.; Sun, D.; Liu, Y.; Fan, Y.; Zhao, L.; Li, X.; Zhu, W. Clinical and high.-resolution CT features of the COVID-19 infection: Comparison of the initial and follow-up changes. Invest. Radiol. 2020. [CrossRef]

9. Chen, G.; Wu, D.; Guo, W.; Cao, Y.; Huang, D.; Wang, H.; Wang, T.; Zhang, X.; Chen, H.; Yu, H. Clinical and immunologic features in severe and moderate Coronavirus Disease 2019. J. Clin. Invest. 2020, 130, 2620-2629. [CrossRef]

10. Tanne, J.H. Covid-19: New York city deaths pass 1000 as Trump tells Americans to distance for 30 days. BMJ 2020, 369, m1333. [CrossRef]

11. Von Elm, E.; Altman, D.G.; Egger, M.; Pocock, S.J.; Gøtzsche, P.C.; Vandenbroucke, J.P. The strengthening the reporting of observational studies in epidemiology (STROBE) statement: Guidelines for reporting observational studies. Int. J. Surg. 2014, 12, 1495-1499. [CrossRef]

12. National Health Commission of the People's republic of China. Diagnosis and Treatment Protocols of Pneumonia Caused by a Novel Coronavirus (6th Version). Available online: http://www.nhc.gov.cn/yzygj/ s7653p/202002/3b09b894ac9b4204a79db5b8912d4440.shtml (accessed on 15 April 2020).

13. Jin, X.; Lian, J.S.; Hu, J.H.; Gao, J.; Zheng, L.; Zhang, Y.M.; Hao, S.R.; Jia, H.Y.; Cai, H.; Zhang, X.L. Epidemiological, clinical and virological characteristics of 74 cases of coronavirus-infected disease 2019 (COVID-19) with gastrointestinal symptoms. Gut 2020, 69, 1002-1009. [CrossRef]

14. Chen, N.; Zhou, M.; Dong, X.; Qu, J.; Gong, F.; Han, Y.; Qiu, Y.; Wang, J.; Liu, Y.; Wei, Y. Epidemiological and clinical characteristics of 99 cases of 2019 novel coronavirus pneumonia in Wuhan, China: A descriptive study. Lancet 2020, 395, 507-513. [CrossRef]

15. Wang, Z.; Yang, B.; Li, Q.; Wen, L.; Zhang, R. Clinical features of 69 cases with Coronavirus disease 2019 in Wuhan. China Clin. Infect. Dis. 2020. [CrossRef] [PubMed]

16. Lescure, F.X.; Bouadma, L.; Nguyen, D.; Parisey, M.; Wicky, P.H.; Behillil, S.; Gaymard, A.; Bouscambert-Duchamp, M.; Donati, F.; Le Hingrat, Q. Clinical and virological data of the first cases of COVID-19 in Europe: A case series. Lancet Infect. Dis. 2020. [CrossRef]

17. Li, K.; Wu, J.; Wu, F.; Guo, D.; Chen, L.; Fang, Z.; Li, C. The clinical and chest CT features associated with severe and critical COVID-19 pneumonia. Invest. Radiol. 2020, 55, 327-331. [CrossRef]

18. Ai, T.; Yang, Z.; Hou, H.; Zhan, C.; Chen, C.; Lv, W.; Tao, Q.; Sun, Z.; Xia, L. Correlation of chest CT and RT-PCR testing in Coronavirus disease 2019 (COVID-19) in China: A report of 1014 cases. Radiology 2020, 200642. [CrossRef] [PubMed]

19. Emanuel, E.J.; Persad, G.; Upshur, R.; Thome, B.; Parker, M.; Glickman, A.; Zhang, C.; Boyle, C.; Smith, M.; Phillips, J.P. Fair allocation of scarce medical resources in the time of Covid-19. N. Engl. J. Med. 2020. [CrossRef]

20. Koonin, L.M.; Pillai, S.; Kahn, E.B.; Moulia, D.; Patel, A. Strategies to inform allocation of stockpiled ventilators to healthcare facilities during a pandemic. Health Secur. 2020, 18, 69-74. [CrossRef]

21. Bhatraju, P.K.; Ghassemieh, B.J.; Nichol, M.; Kim, R.; Jerome, K.R.; Nalla, A.K.; Greninger, A.L.; Pipavath, S.; Wurfel, M.M.; Evans, L. Covid-19 in critically Ill patients in the seattle region-case series. N. Engl. J. Med. 2020. [CrossRef]

22. Zhang, X.; Cai, H.; Hu, J.; Lian, J.; Gu, J.; Zhang, S.; Ye, C.; Lu, Y.; Jin, C.; Yu, G. Epidemiological, clinical characteristics of cases of SARS-CoV-2 infection with abnormal imaging findings. Int. J. Infect. Dis. 2020, 94, 81-87. [CrossRef] 
23. Liu, Y.; Yan, L.M.; Wan, L.; Xiang, T.X.; Le, A.; Liu, J.M.; Peiris, M.; Poon, L.L.M.; Zhang, W. Viral dynamics in mild and severe cases of COVID-19. Lancet Infect. Dis. 2020. [CrossRef]

24. Wolfel, R.; Corman, V.M.; Guggemos, W.; Seilmaier, M.; Zange, S.; Müller, M.A.; Niemeyer, D.; Jones, T.C.; Vollmar, P.; Rothe, C. Virological assessment of hospitalized patients with COVID-2019. Nature 2020. [CrossRef] [PubMed]

25. Grieser, C.; Goldmann, A.; Steffen, I.G.; Kastrup, M.; Fernández, C.M.; Engert, U.; Deja, M.; Lojewski, C.; Denecke, T. Computed tomography findings from patients with ARDS due to Influenza A (H1N1) virus-associated pneumonia. Eur. J. Radiol. 2012, 81, 389-394. [CrossRef] [PubMed]

26. Sheshadri, A.; Shah, D.P.; Godoy, M.; Erasmus, J.J.; Song, J.; Li, L.; Evans, S.E.; Chemaly, R.F.; Dickey, B.F.; Ost, D.E. Progression of the radiologic severity index predicts mortality in patients with parainfluenza virus-associated lower respiratory infections. PLoS ONE 2018, 13, e0197418. [CrossRef] [PubMed]

(C) 2020 by the authors. Licensee MDPI, Basel, Switzerland. This article is an open access article distributed under the terms and conditions of the Creative Commons Attribution (CC BY) license (http://creativecommons.org/licenses/by/4.0/). 